\title{
A Novel Lactiferous Duct Preserving Method for Inverted Nipples: An Inlay Wrap-Around Flaps Supporting the Nipple Column
}

Jun Ho Park, Syeo Young Wee, Hyun Gyo Jeong,

Chang Yong Choi

Department of Plastic and Reconstructive Surgery, Soonchunhyang Gumi Hospital, Soonchunhyang University College of Medicine, Gumi, Korea
No potential conflict of interest relevant to this article was reported.
Background Inverted nipples can pose aesthetic and functional problems, especially for young women. The objectives of inverted nipple correction are both sufficient aesthetic projection of the nipple and postoperative functional preservation of the lactiferous ducts. Recurrence of nipple inversion is still an unsolved problem in many cases. We present a new nipple suspension technique using nipple- and areola-based dermal flaps for correcting inverted nipples and preserving the lactiferous duct to minimize the risk of recurrence.

Methods We corrected six inverted nipples in three patients, which were classified as grade II using the Han and Hong classification of nipple inversion. The anteroposterior and lateral medical-quality photographs of the respective patients were identified for nipple protrusion and recurrence during a 6-month follow-up period to evaluate aesthetical goal and assess the sensitivity of the nipple to confirm preservation of the main lactiferous ducts. Surgical details are described within the main text.

Results Preoperative and postoperative photography revealed good nipple protrusion during the 6-month follow-up period without any complications such as skin necrosis and recurrence. We were unable to directly identify breast-feeding function because all three patients were young and unmarried women. However, we assumed preservation of the main lactiferous duct since no nipple sensory change was identified in the postoperative examination when compared with the preoperative examination. Conclusions With this method, we were able to confirm the hardness of the column and minimize the injury of the main lactiferous duct.

Keywords Breast, Lactiferous duct, Nipples, Recurrence, Surgical flaps

\section{INTRODUCTION}

An inverted nipple is a condition in which the nipple is buried below the plane of the areola, characterized by short galactophorous ducts, periductal fibrosis, and lack of soft tissue under the nipple

Received: Nov 7, 2016 Revised: Jan 13, 2017 Accepted: Jan 13, 2017 Correspondence: Syeo Young Wee Department of Plastic and Reconstructive Surgery, Soonchunhyang University Gumi Hospital, 179 1 Gongdan-ro, Gumi 39371, Korea. E-mail: ouisy@naver.com

Copyright () 2017 The Korean Society for Aesthetic Plastic Surgery.

This is an Open Access article distributed under the terms of the Creative Commons Attribution Non-Commercial License (http://creativecommons.org/licenses/by-nc/4.0/) which permits unrestricted non-commercial use, distribution, and reproduction in any medium, provided the original work is properly cited. $\quad w w w . e-a a p s . o r g$ base. This deformity is a relatively common problem, with reported prevalence ranging from 1.8 to $3.3 \%$ [1]. Inverted nipples can pose aesthetic and functional problems, especially for young women. The objectives of inverted nipple correction are both sufficient aesthetic projection of the nipple and postoperative functional preservation of the lactiferous ducts. Over time, surgeons have used various areolar dermal flap techniques to correct this condition. However, recurrence of nipple inversion is still an unsolved problem in many cases.

In this article, we present a new nipple suspension technique using nipple- and areola-based dermal flaps for correcting inverted nipples and preserving the lactiferous duct to minimize the risk of recurrence. 

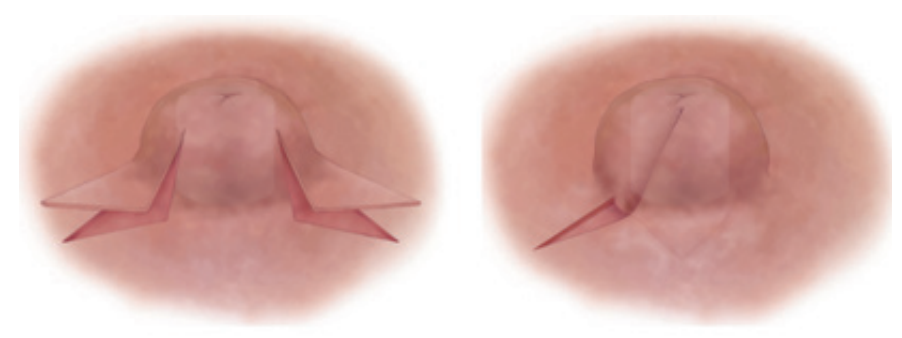

Fig. 1. Schematic illustration of operative procedure.

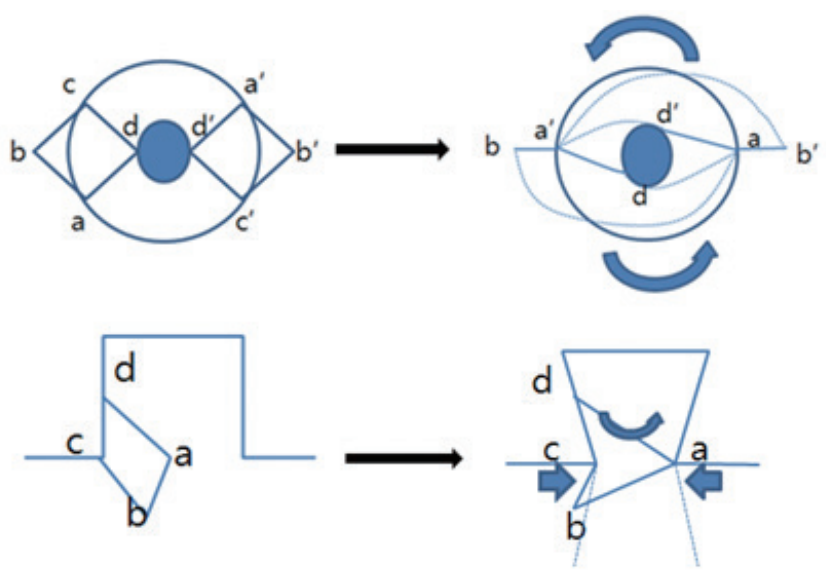

Fig. 2. Schematic diagram of operative procedure.

\section{METHODS}

We corrected six inverted nipples in three patients, which were classified as grade II using the Han and Hong classification of nipple inversion [2]. All of the records and information of the patients were anonymized and de-identified prior to analysis. The anteroposterior and lateral medical-quality photographs of the respective patients were identified for nipple protrusion and recurrence during a 6-month follow-up period to evaluate aesthetical goal and assess the sensitivity of the nipple to confirm preservation of the main lactiferous ducts.

\section{Surgical technique}

Two diamond-shaped flaps were designed at the 3- and 9-oclock positions of the nipple. Each flap was placed between the areola and the nipple (Fig. 1 and 2).The flaps were elevated according to the edges of the nipple portion. During division, we released the retractile ducts and the fibrous bands of the circumferential side of the nipple, preserving the main ducts. Then, the elevated flaps were wrapped around the nipple columns in the counter-clockwise direction, resulting in reduction of the nipple neck. The lower portions of the flaps were flexibly designed as an inset to fill the dead space and to wrap the column.

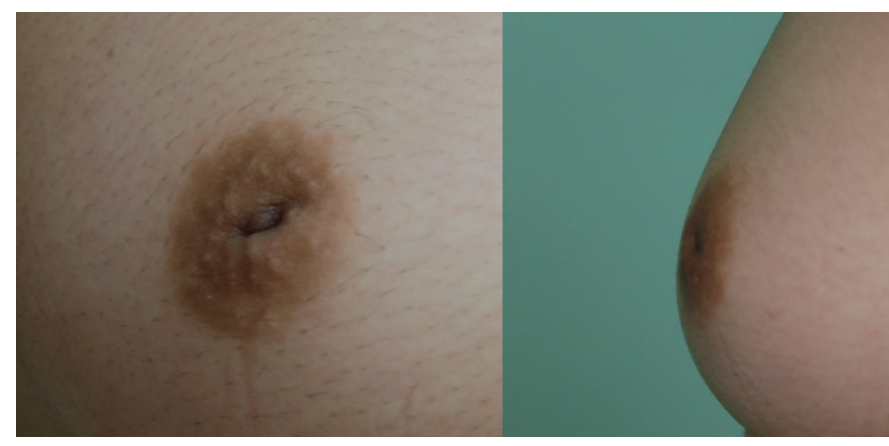

Fig. 3. Preoperative photographic finding.

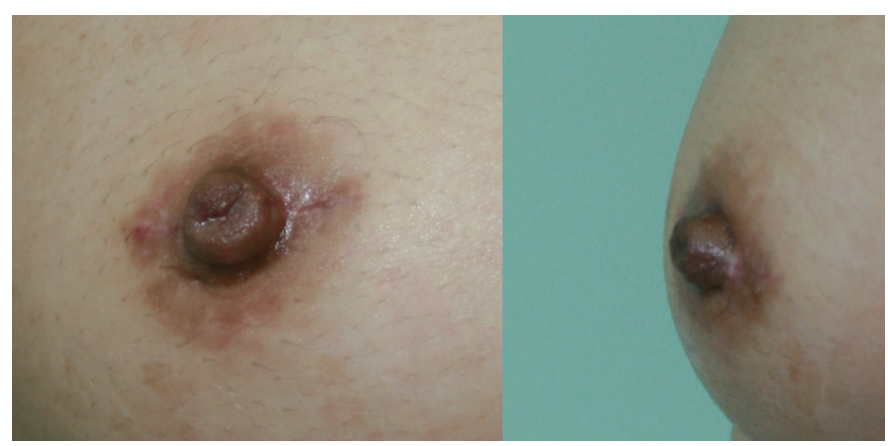

Fig. 4. Postoperative photographic finding.

\section{RESULTS}

Preoperative and postoperative photography revealed good nipple protrusion during the 6-month follow-up period without any complications such as skin necrosis and recurrence (Fig. 3 and 4). Neither postoperative traction suture nor special dressing was required because of strong suspension of the nipple column.

In this study, we were unable to directly identify breast-feeding function because all three patients were young and unmarried women. However, we assumed preservation of the main lactiferous duct since no nipple sensory change was identified in the postoperative examination when compared with the preoperative examination.

\section{DISCUSSION}

According to the Han and Hong classification [3], Grade II inverted nipples have moderate underlying fibrosis, and they are difficult to pull out manually. Despite traction pressure on the nipple to force it to protrude, it would promptly retract. Therefore, many surgeons have difficulty in obtaining an optimal release of the fibrotic band with preservation of the ducts, leading to nipple inversion or flattening recurrence.

Corrective surgical procedures of inverted nipples have two basic objectives. First, adequate release of periductal fibrous tissues and secondly, preservation of the lactiferous duct. 
In most of the previously introduced techniques, areolar dermal flap techniques were used to fill the dead space underneath the nipple that was developed after sufficient release of periductal fibrous tissues. Elsahy $[4,5]$ originally proposed the use of bilateral triangular dermal flaps that cross under the nipple. Due to its simplicity and effectiveness, this method became widely used in both its original and modified forms [2,6-9]. However, it is difficult to provide a rigid fulcrum with those techniques, and the main lactiferous duct could be injured by inserting a flap. In a recent procedure [9], the base of the nipple was supported by raising the diamond-shaped areolar dermal flap and crossing over to the opposite side subcutaneously through a nipple tunnel. However, this 'areolar based' flap was not easy to rotate and resulted in nipple torsion during suturing.

For the proposed modification of the areolar dermal flap technique, we placed the axis of the flap at the nipple portion rather than the areola to minimize nipple torsion. Our focus was to reduce the nipple stalk diameter by transverse suturing on the opposite side and to improve nipple projection by maintaining nipple volume using the upper portion of the flap. The lower flap was used to fill the dead space resulting from release of the fibrous band.

By utilizing the blood supply of only one single edge of the flap, limited circulation would be possible for the lower flap. However, the main blood supply of the nipple areolar complex is derived from the external and internal mammary arteries that transverse subcutaneous tissues. As nutrient vessels in the subcutaneous tissues supply the nipple, we could preserve the blood supply of the nipple-areolar complex transposition flap based on the dermal pedicle [10]. In our study, design of the random flap based on the dermal plexus did not exceed areolar tissue boundary; thus, there was no problem associated with blood circulation.

Another important aspect to consider in inverted nipple correction surgery is lactation. In our procedure, a limited size of the dermal flap was buried and anchored to maintain lactation [11]. The nipple is innervated from the lateral cutaneous branch of the fourth intercostal nerve along the major duct system [12]. The lactiferous duct is loosely encircled by small and large nerve fibers that form whorls from the lactiferous sinus to the nipple tip [13]. To minimize duct injury, we released periductal fibrosis tissues and maintained the central portion of the stalk in which the main the lactiferous duct is located. If correction was found to be insufficient, a subcutaneous tunnel was made by repeated vertical splitting maneuvers with fine Metzenbaum dissecting scissors, which minimized main duct injury. All of our patients maintained good nipple sensation, suggesting preservation of the main duct.

This study has several limitations. First, the shape of the nipple could appear embossed in the short-term follow-up period. A narrowed nipple neck and flap insertion at both the upper and lower portion of the nipple induced postoperative shape deformity. How- ever, in the 6-month follow-up period, the shape became flattened and normalized. In addition, the number of cases was too small; thus, more cases are needed to reach a universal conclusion.

\section{CONCLUSIONS}

We designed a diamond-shaped nipple-areolar based dermal flap to correct moderately inverted nipples. In this study, nipple protrusion was aesthetically acceptable in all patients postoperatively. Unlike procedures introduced by other surgeons previously, we set the axis of the flap at the nipple portion and wrapped stalk. In addition, nipple stalk diameter was reduced, and we were able to increase nipple volume and fill the dead space in the lower portion of the nipple.

With this method, we were able to confirm the hardness of the column and minimize the injury of the main lactiferous duct. Therefore, this technique would be efficient in the treatment of moderately inverted nipples and can be considered as an option for surgeons who commonly deal with this pathology.

\section{PATIENT CONSENT}

Patients provided written consent for the use of their images.

\section{REFERENCES}

1. Park HS, Yoon CH, Kim HJ. The prevalence of congenital inverted nipple. Aesthetic Plast Surg 1999;23:144-6.

2. Haeseker B. The application of de-epithelialised "turn-over" flaps to the treatment of inverted nipples. Br J Plast Surg 1984;37:253-5.

3. Han S, Hong YG. The inverted nipple: its grading and surgical correction. Plast Reconstr Surg 1999;104:389-95; discussion 96-7.

4. Elsahy NI. An alternative operation for inverted nipple. Plast Reconstr Surg 1976;57:438-91.

5. Elsahy N. Correction of inverted nipples by strong suspension with areola-based dermal flaps. Plast Reconstr Surg 2009;123:1131.

6. Teimourian B, Adham MN. Simple technique for correction of inverted nipple. Plast Reconstr Surg 1980;65:504-6.

7. Lee HB, Roh TS, Chung YK, et al. Correction of inverted nipple using strut reinforcement with deepithelialized triangular flaps. Plast Reconstr Surg 1998;102:1253-8.

8. Kim DY, Jeong EC, Eo SR, et al. Correction of inverted nipple: an alternative method using two triangular areolar dermal flaps. Ann Plast Surg 2003;51:636-40.

9. Burm JS, Kim YW. Correction of inverted nipples by strong suspension with areola-based dermal flaps. Plast Reconstr Surg 2007;120:1483-6.

10. Nakajima H, Imanishi N, Aiso S. Arterial anatomy of the nipple-areola complex. Plast Reconstr Surg 1995;96:843-5.

11. Kim JH, Ahn HC. A revision restoring projection after nipple reconstruction by burying four triangular dermal flaps. Arch Plast Surg 2016; 
43:339-43.

12. Schlenz I, Kuzbari R, Gruber H, et al. The sensitivity of the nipple-areola complex: an anatomic study. Plast Reconstr Surg 2000;105:905-9.
13. Montagna W, Macpherson EE. Proceedings: Some neglected aspects of the anatomy of human breasts. J Invest Dermatol 1974;63:10-6. 